\title{
Recrystallization of Water in a Non-Water-Soluble Polymer Examined by Fourier Transform Infrared Spectroscopy: Poly(2-methoxyethylacrylate) with Low Water Content
}

\author{
Makoto Gemmei-Ide* and Hiromi Kitano* \\ Department of Environmental Applied Chemistry, Graduate School of Science and Engineering, \\ University of Toyama, Gofuku 3190, Toyama 930-8555, Japan
}

Tel: +81-76-445-6868; Fax: +81-76-445-6703

e-mail: (H.K.) kitano@eng.u-toyama.ac.jp; (M.G.-I.) ide@eng.u-toyama.ac.jp

\begin{abstract}
Crystallization of water during heating, so-called "recrystallization of water", in poly(2-methoxylethylacrylate) (PMEA) was investigated by temperature-variable Fourier transform infrared spectroscopy. Recrystallization in a polymer-water system is generally understood to be phase transition from glassy water (condensed water) to crystalline water. However, infrared spectral changes of the PMEA-water system with low water content indicated that the formation of ice $I_{\mathrm{h}}$ during heating occurred by a vapor deposition process rather than by a crystallization process.
\end{abstract}

\section{Key Words}

Infrared Spectroscopy / Phase Transition / Recrystallization / Solid Polymer / Water

Crystallization of water occurs not only during cooling but also during heating: the latter is frequently called "recrystallization" (or "cold crystallization"). Recrystallization temperature, $T_{\mathrm{R}}$, of water (hyperquenched glassy water, HQGW) has been reported to be $\sim 150 \mathrm{~K} .{ }^{1}$ Aqueous solutions are also recrystallizable and their $T_{\mathrm{R}} \mathrm{s}$ are usually higher than that of HQGW. In the case of a high concentration, recrystallization can be easily observed without a special technique such as preparation of $\mathrm{HQGW}^{1,2}$ in various aqueous solutions such as glycerol, sucrose, polyvinylpyrrolidone, and poly(ethylene glycol) aqueous solutions. ${ }^{3}$ The most cases reported are aqueous solutions, i.e., binary systems composed of a water-soluble solute and water.

Recrystallization of water in a non-water-soluble polymer, poly(2-methoxyethylacrylate) (PMEA), was recently demonstrated by Tanaka et al. using differential scanning calorimetry. ${ }^{4}$ They reported that recrystallization of water in PMEA occurred in the water content region of 3.0-9.0 wt $\%$ at $\sim 220 \mathrm{~K}$, and they called the recrystallized water "intermediate water" or "freezing bound water". 4,5 Intermediate water is water having a specialized structure of a hydrogen-bonding network at the physiological temperature. PMEA, on the other hand, is also known to be an excellent platelet compatible material. ${ }^{6}$ The research group of Tanaka concluded that intermediate water played an important role in the expression of platelet compatibility. ${ }^{5}$

The aim of this study was not to clarify the structure of recrystallizable water and the correlation of platelet compatibility with water structure. The primary goal was to determine whether recrystallization of water in a non-water-soluble polymer is physicochemically the same as that in aqueous solutions. A general explanation for recrystallization in aqueous solutions is that glassy water, i.e., solid water without crystalline form, crystallizes during heating. ${ }^{3}$ Recrystallization of water in a PMEA matrix has also been recognized to be a phase transition from glassy water to ice during heating. ${ }^{4,5}$

In this study, recrystallization of water in a PMEA matrix was investigated by Fourier transform infrared spectroscopy. Different to the generally accepted process of recrystallization in an aqueous solution, results of infrared spectroscopy clearly indicated that the majority of recrystallized water in a PMEA matrix was not formed from glassy water. The term "re-vapor-deposition" might be more appropriate than the term "recrystallization" for the process of ice formation in PMEA during heating.

The spectrum of water sorbed into PMEA (water content of $3.2 \mathrm{wt} \%$ ) at $298 \mathrm{~K}$ is shown in Figure 1. A comparison with liquid water indicates that the majority of sorbed water might be water hydrated to the polymer chain via hydrogen bonding (HB): liquid water has a main peak at $3400 \mathrm{~cm}^{-1}$ and a shoulder at $3250 \mathrm{~cm}^{-1}$, whereas the sorbed water has a main peak at $3593 \mathrm{~cm}^{-1}$ and three shoulders at 3500,3420 , and $3280 \mathrm{~cm}^{-1}$. The higher two and lower two components might be attributed to water hydrated to ester and ether groups, respectively. ${ }^{8}$ At present, it is not clear whether the lower two components include a component from condensed water, but even if it exists, its amount should be small because the absorbtivity of fully-hydrogen-bonded water is greatly enhanced compared with that of monomolecular water. ${ }^{9}$ 
The spectral responses of sorbed water by a thermal perturbation $(298 \mathrm{~K} \leftrightarrow 170 \mathrm{~K})$ at cooling rates of 5.0 and $0.25 \mathrm{~K} \cdot \mathrm{min}^{-1}$ are shown in Figures 2 and 3, respectively. Heating rate for the spectral responses in both figures was $0.5 \mathrm{~K} \cdot \mathrm{min}^{-1}$. Figure 4 shows results for pure water at cooling and heating rates of $0.5 \mathrm{~K} \cdot \mathrm{min}^{-1}$. The values of $A_{3590}$ and $A_{3280}$ (bottom panels in the figures) are used as indicators for monomolecular water hydrated to the polymer chain and condensed water (ice $I_{\mathrm{h}}$ ), respectively. The value of $A_{3280}$ might have a double origin (the hydrated water and condensed water) as mentioned above, which indicates that the value of $A_{3280}$ cannot directly give the exact ratio of the two types of water. However, as seen in the case of pure water (Figure 4), the ice formation can be decided from the discontinuous change in the value of $A_{3280}$ at phase transition and the appearance of a shoulder centered at $3150 \mathrm{~cm}^{-1}$.

Rapid Cooling (5.0 K $\cdot \mathbf{m i n}^{-\mathbf{1}}$, Figure 2). In the cooling process, the absorptive intensities in the lower wavenumber region nonlinearly increased as seen in the contour map and the value of $A_{3280}$. The spectra of sorbed water in all $T$ regions were quite different to ice $I_{\mathrm{h}}$, which showed no ice formation. The nonlinear increase indicates a change in relative ratios of the hydration species. This is supported by a synchronism of nonlinear decrease in the value of $A_{3590}$.

In the heating process, a complex change occurred. In two $T$ regions of 170-211 K and 267-298 K, the spectra were traceable with those in the cooling process. In the $T$ region of $211-267 \mathrm{~K}$, an ice-like spectrum appeared. This is the so-called "recrystallization of water". A general explanation for this is that glassy water formed by rapid cooling crystallizes upon heating. ${ }^{3-5}$ However neither spectra for glassy water ${ }^{10}$ nor spectra for low-density liquid water ${ }^{11}$ were obtained. Moreover, the value of $A_{3280}$ at $235 \mathrm{~K}$ might be too much large even if liquid water existed at $298 \mathrm{~K}$ and this water recrystallized. The rate of increase in $A_{3280}$ of pure water from $298 \mathrm{~K}$ (liquid) to $235 \mathrm{~K}$ (ice) is only $267 \%$ (Figure 4), whereas it is $720 \%$ in the present case. This much larger increase can be explained by crystal growth via the deposition of water monomolecularly dispersed in the polymer matrix. In other words, the ice formed by recrystallization in PMEA is not generated from glassy water. The synchronized change in $A_{3590}$ supports this assumption. Crystal growth (increase in $A_{3280}$ ) began at $211 \mathrm{~K}$, suggesting that the diffusion of sorbed water was suppressed below $211 \mathrm{~K}$.

Slow Cooling $\left(\mathbf{0 . 2 5} \mathrm{K} \cdot \mathrm{min}^{-1}\right.$, Figure 3). In contrast to the above case, a drastic change occurred during cooling: the spectral profile in the lower wavenumber region below $211 \mathrm{~K}$ was just that of ice $I_{\mathrm{h}}$. The value of $A_{3280}$ gradually increased and the rate of increase abruptly became large at $230 \mathrm{~K}$ and then became small below $211 \mathrm{~K}$. This indicates that a part of sorbed water freezes below $230 \mathrm{~K}$. As in the case of recrystallization after rapid cooling, the increase in $A_{3280}$ synchronized with the decrease in $A_{3590}$, suggesting that crystallization and recrystallization were the same, i.e., association of monomolecular water. In addition, the rate of increase in $A_{3280}$ of $114 \%$ from $211 \mathrm{~K}$ to $170 \mathrm{~K}$ was very similar to that of ice, $112 \%$. Thus, the increase in $A_{3280}$ below $211 \mathrm{~K}$ does not indicate crystal growth of ice but $T$ dependence of $A_{3280}$ of ice and suggests again suppression of the diffusion of water molecules below $211 \mathrm{~K}$.

In the heating process, recrystallization occurred again. Starting $T$ of it was $211 \mathrm{~K}$, the same as that at the rapid cooling rate The manner of change in $A_{3590}$ was also the same: it synchronized with the increase in $A_{3280}$. These results show that the recrystallization observed here also is the association of monomolecular water and that this recrystallized water might correspond to water remained as monomolecular water, which does not crystallize due to the faster cooling rate than the condensing rate. In the case of rapid cooling, most of the sorbed water might be remaining water. Therefore, if cooling rate is very slow, no recrystallization of water should occur.

Vapor Deposition and Re-vapor-deposition. As stated above, the ice formation in the PMEA matrix is induced by the association of monomolecular water molecules. Thus, "crystallization" or "recrystallization" might not be a suitable term for the process of ice formation in a PMEA matrix because the two terms generally signify phase transition from condensed fluid (including glass) to crystalline. Therefore, at least when the water content is low as in the present case, the term "vapor deposition" or "re-vapor-deposition" might be more suitable.

Vapor deposition and re-vapor-deposition $T$ s were $230 \mathrm{~K}$ and $211 \mathrm{~K}$, respectively. The reason for re-vapor-deposition $T$ of $211 \mathrm{~K}$ might be suppression of the diffusion of water molecules. This $T$ might correspond to a balancing point between dissociation force from the polymer chain by the diffusion and restriction force by the HB. This $T$ is the same as the temperature at which the change in $A_{3280}$ during cooling became linearly related to $T$, strongly supporting the diffusion suppression.

Glass transition of the PMEA-water system should be mentioned here because the water diffusion in a polymer solid might be affected by movement of the polymer chain. $T_{\mathrm{g}}$ values of PMEA-water systems $(0.2-36.0$ wt $\%$ of water) have been reported to be $\sim 223 \mathrm{~K}$, and starting $T$ s of glass-to-rubber and rubber-to-glass transitions have been reported to be $\sim 220 \mathrm{~K}$ and $\sim 232 \mathrm{~K}$, respectively. ${ }^{4}$ These temperatures are much higher than re-vapor-deposition $T, 211 \mathrm{~K}$. This fact indicates no relation of movement of the polymer chain to re-vapor-deposition. The vapor deposition $T(230 \mathrm{~K})$ is, on the other hand, very close to the starting $T$ of rubber-to-glass transition, $232 \mathrm{~K}$. In many cases, the activation energy of diffusion of a sorbate in a polymer matrix becomes low at temperatures below $T_{\mathrm{g}}{ }^{12}$ suggesting that movement of the polymer chain spatially (sterically) prevents the diffusion of sorbate and might prevent the self-association of sorbates. The association tendency of sorbed water, on the other hand, increases with decrease in temperature, which should be a thermodynamic requirement for any fluid. As a result, when the polymer drops into a glassy state, the water association might be accelerated. In the case of PMEA, the starting $T$ of rubber-to-glass transition is lower than homogeneous nucleation $T$ of normal water, $236 \mathrm{~K} .{ }^{13}$ In this situation, the associated water might immediately crystallize when the number of associated water molecules becomes sufficient to form an ice nucleus by overcoming the water-polymer interfacial energy. After the ice nucleus has been formed, ice $I_{\mathrm{h}}$ might be grown by vapor deposition until $211 \mathrm{~K}$. The reason for the starting $T$ of re-vapor-deposition and ending $T$ of vapor deposition (crystal growth) being the same can be well explained by suppression of the diffusion of water molecules. 
In summary, so-called recrystallization of water in a PMEA matrix with a low water content was investigated using a vibrational spectroscopic method, which clearly indicated that the term "re-vapor-deposition" was more suitable than the term "recrystallization" for the process of ice formation during heating. Furthermore, the slow cooling induced the formation of ice during cooling, a process for which the term "vapor deposition" was also more suitable than the term "crystallization". Starting temperatures of re-vapor-deposition and vapor deposition might be governed by the diffusion properties of water. The former might occur when the diffusion force of water molecules becomes larger than the restriction force by HB, and the latter might occur when the motion of the polymer chain is frozen during cooling. These processes of ice formation are clearly different from the general explanation for recrystallization/crystallization in concentrated aqueous solutions. ${ }^{3}$ However, such ice formation by re-vapor-deposition and vapor deposition in polymer solids might not be strange if we accept the following two assumptions: (1) water molecules sorbed into polymer solids tend to associate with each other by cooling, i.e., the association state of water is drastically changed by temperature, and (2) suppression of the diffusion of water molecules occurs at temperature where the restriction force by $\mathrm{HB}$ overcomes the dissociation force by diffusion of water molecules.

Acknowledgments. This work is supported by a Grant-in-Aid for Young Scientists (B: 20750175) from the Ministry of Education, Culture, Sports, Science and Technology of Japan and a Grant-in-Aid for Scientific Research (B: 19350055) from the Japan Society for the Promotion of Science.

Supporting Information Available: Experimental methods. This information is available free of charge via the Internet at http://pubs.acs.org.

\section{References and Notes}

(1) (a) Brüeggeller, P.; Mayer, E.; Nature 1980, 288b, 569. (b) Brüeggeller, P.; Mayer, E.; Nature 1982, $298,715$.

(2) Kim, W.-H.; McPhillen, M.; Hayes, J. M.; Small, G. J. Chem. Phys. Lett. 1983, 207, 443.

(3) (a) MacKenzie, A. P. Phil. Trans. R. Soc. Lond. B. 1977, 278, 167. (b) Franks, F. In Water: A Comprehensive Treatise; Franks, F., Ed.; Plenum Press: New York, 1982; Vol. 7, pp 215-338.

(4) Tanaka, M.; Motomura, T.; Ishii, N.; Shimura K.; Onishi, M.; Mochizuki, A.; Hatakeyama, T. Polym. Int. $2000,49,1709$.

(5) (a) Tanaka, M.; Mochizuki, A.; Ishii, N.; Motomura, T.; Hatakeyama, T. Biomacromolecules 2002, 3, 36. (b) Tanaka, M. and Mochizuki, A. J. Biomed. Mater. Res. A 2004, 78A, 684. (c) Hirota E.; Tanaka, M.; Mochizuki A. J. Adv. Sci. 2005, 17, 245.

(6) Tanaka, M.; Motomura, T.; Anzai T.; Kasori Y.; Onishi, M.; Shiroya, T.; Shimura, K.; Mochizuki, A. Biomaterials 2000, 21, 1471 .

(7) The term "re-vapor-deposition" means vapor deposition during heating and is an imitation of the term "recrystallization". This is to avoid confusion with the term "vapor redeposition" frequently used in the field of solid surface processing via vapor deposition.

(8) (a) Kusanagi, H.; Yukawa, S. Polymer 1994, 35, 5637. (b) Ichikawa, K.; Mori, T.; Kitano, H.; Fukuda, M.; Mochizuki, A.; Tanaka, M. J. Polym. Sci. Polym. Phys. 2001, 39, 2175. (c) Gemmei-Ide, M.; Motonaga T.; Kitano, H. Langmuir 2006, 22, 2242. (d) Morita, S.; Tanaka, M.; Ozaki, Y. Langmuir 2007, 23, 3750.

(9) (a) Eisenberg, D.; Kauzmann, W. In The Structure and Properties of Water; Clarendon Press: London, 1969; Chapter 4. (b) Fukuda, M. JCPE J. 2001, 13, 105.

(10) Givan, A.; Loewenschuss, A.; Nielsen, C. J. J. Phys. Chem. B. 1997, 101, 8696.

(11) Mallamace, F.; Broccio, M.; Corsaro, C.; Faraone, A.; Majolino, D.; Venuti, V.; Liu, L.; Mou, C.Y.; Chen, S.H. Proc. Natl. Acad. Sci. USA 2005, 104, 424.

(12) Takizawa, A.; Hamada, T.; Okada, H.; Kadota, S. Polymer 1974, 15, 157.

(13) (a) Hobbs, P. V. In Ice Physics; Clarendon Press: Oxford, U.K., 1974; pp 461-523. (b) Angell, C. A. In Water: A Comprehensive Treatise; Franks, F., Ed.; Plenum Press: New York, 1982; Vol. 7, pp 1-81.

\section{Figure Captions}

Figure 1. Infrared spectra for pure water (dotted line) and water sorbed into PMEA with a water content of $3.2 \mathrm{wt} \%$ at $298 \mathrm{~K}$.

Figure 2. Temperature-dependence of infrared spectra for water sorbed into PMEA at a cooling rate of $5.0 \mathrm{~K} \cdot \mathrm{min}^{-1}(\mathrm{left})$ and at a heating rate of $0.5 \mathrm{~K} \cdot \mathrm{min}^{-1}$ (right). Top, original spectra; middle, contour map; bottom, $T$-dependence of $A_{3590}$ and $A_{3280}$.

Figure 3. Temperature-dependence of infrared spectra for water sorbed into PMEA at a cooling rate of $0.25 \mathrm{~K} \cdot \mathrm{min}^{-1}(\mathrm{left})$ and at a heating rate of $0.5 \mathrm{~K} \cdot \mathrm{min}^{-1}$ (right). Top, original spectra; middle, contour map; bottom, $T$-dependence of $A_{3590}$ and $A_{3280}$.

Figure 4. Temperature-dependence of infrared spectra for pure water at a cooling rate of $0.5 \mathrm{~K} \cdot \mathrm{min}^{-1}$ (left) and at a heating rate of $0.5 \mathrm{~K} \cdot \mathrm{min}^{-1}$ (right). Top, original spectra; bottom, $T$-dependence of $A_{3280}$. 


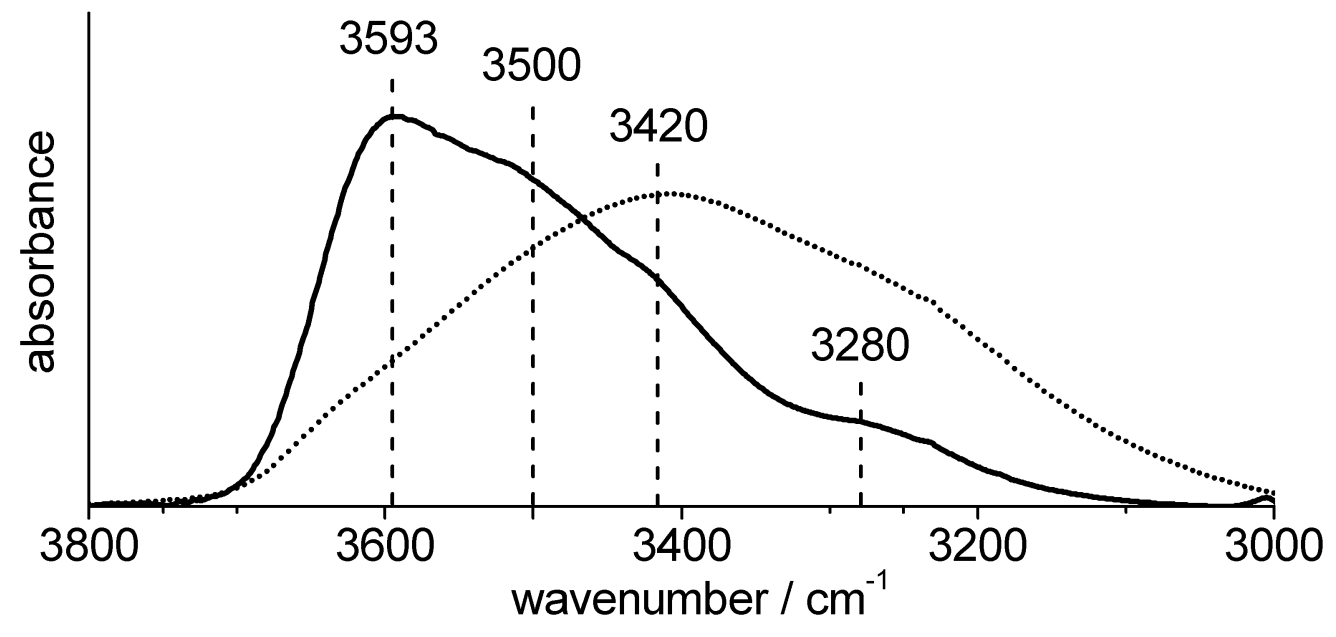

Figure 1. Gemmei-Ide \& Kitano 

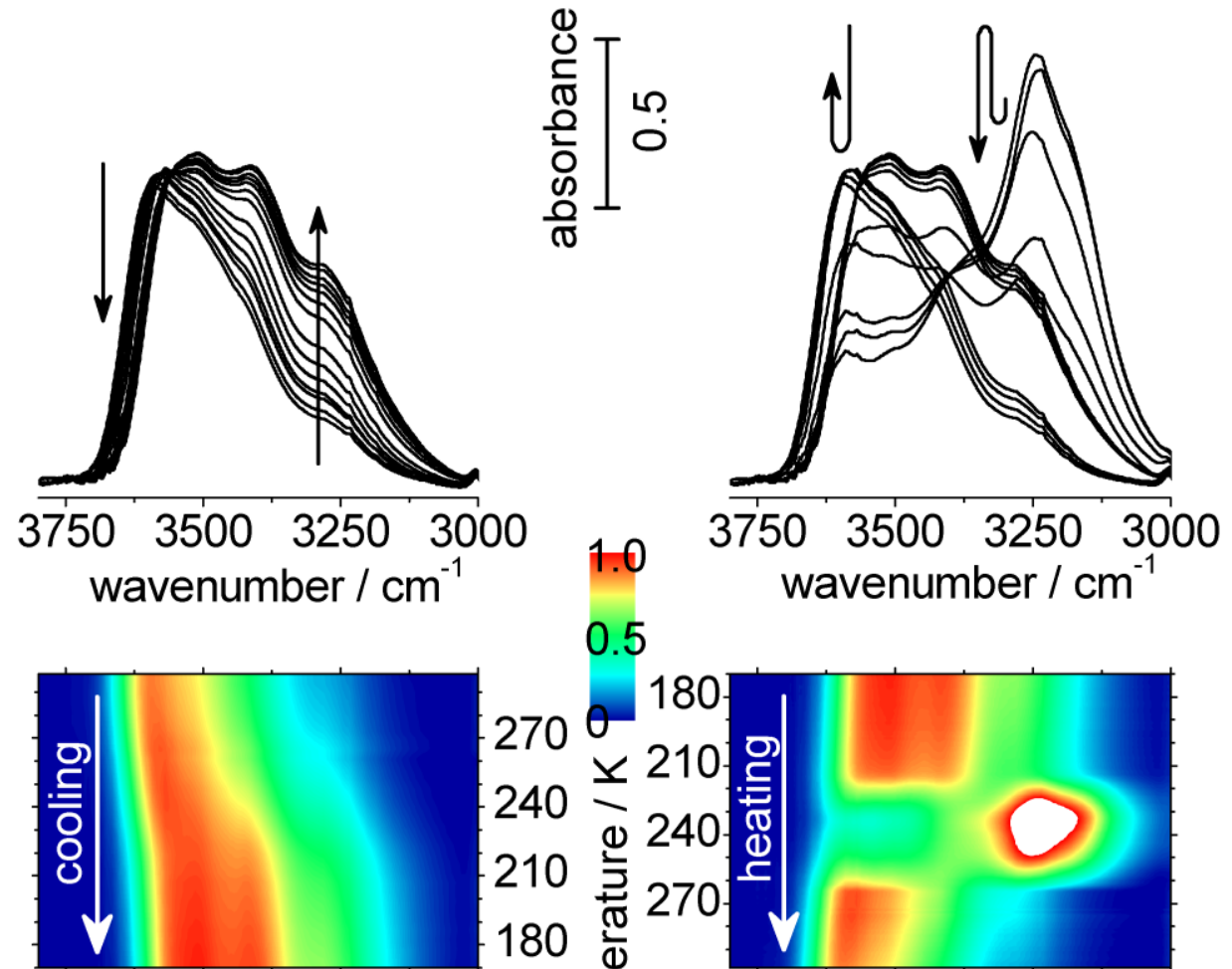

$3750 \quad 3500 \quad 3250 \quad 3000$ wavenumber $/ \mathrm{cm}^{-1}$

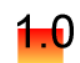

0.5

270

180
$\square 210$

240

210

离 240

$\frac{\pi}{\frac{\pi}{2}} 270$

$\stackrel{\circ}{\&} \quad 3750 \quad 350032503000$
$\stackrel{\Phi}{+} \quad$ wavenumber $/ \mathrm{cm}^{-1}$
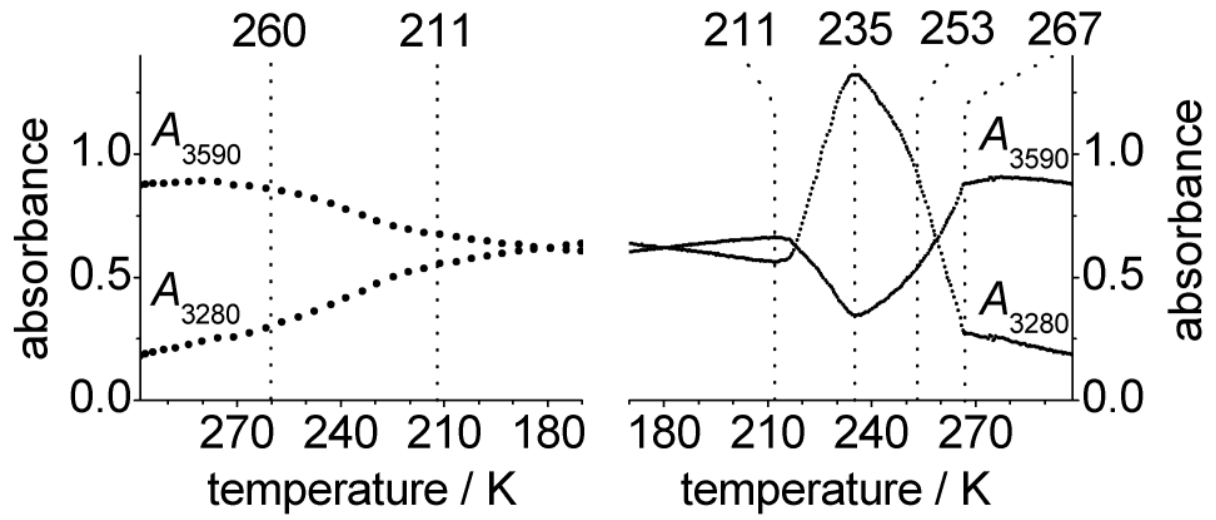

Figure 2. Gemmei-Ide \& Kitano 

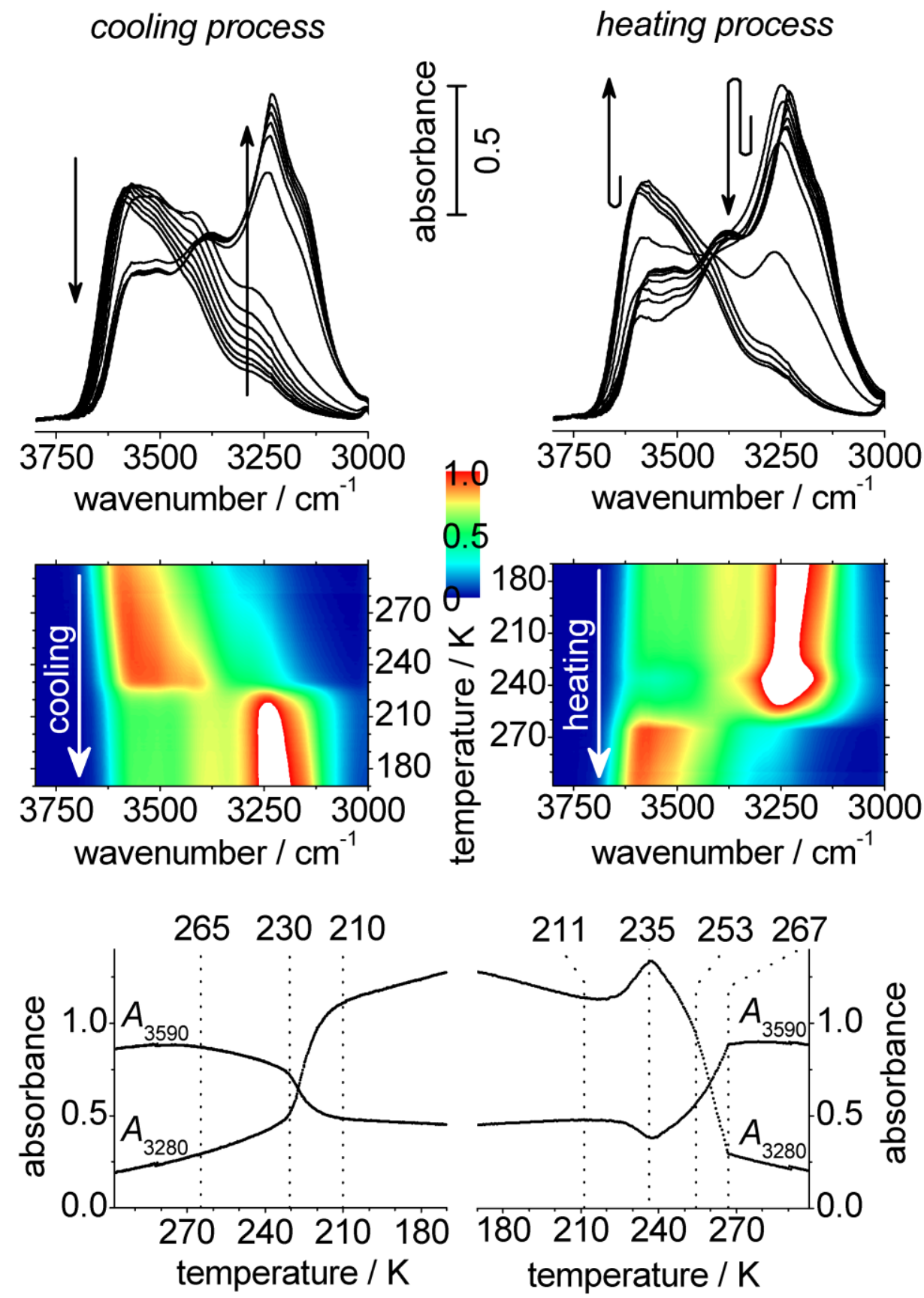

Figure 3. Gemmei-Ide \& Kitano 

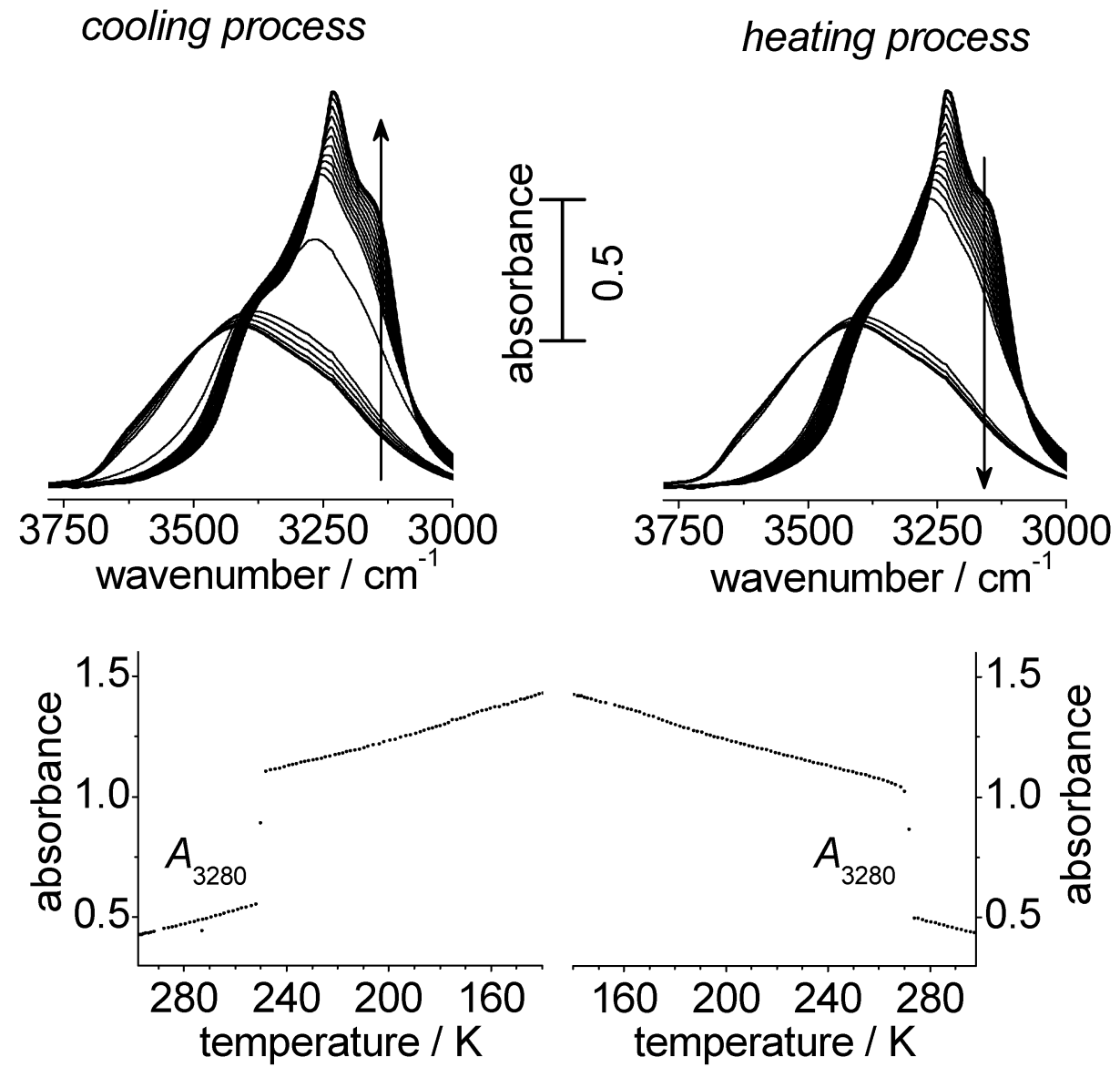

Figure 4. Gemmei-Ide \& Kitano 


\section{TOC Graphic}

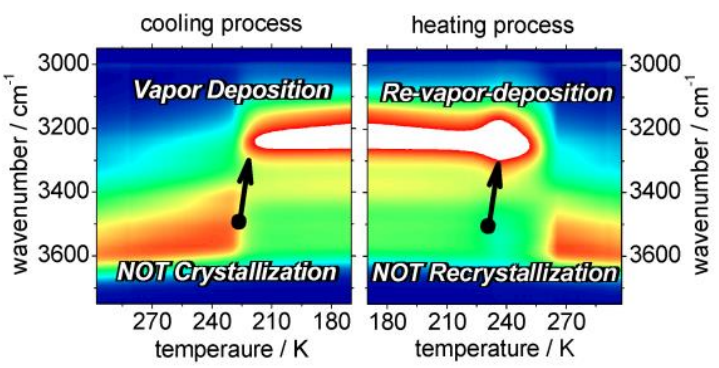

